\title{
Value of cardiac biomarker measurement in the differential diagnosis of infiltrative cardiomyopathy patients with preserved left ventricular systolic function
}

\author{
Kai Hu ${ }^{1 \#}$, Dan Liu ${ }^{1 \#}$, Tim Salinger ${ }^{1}$, Daniel Oder ${ }^{1}$, Stefan Knop $^{2}$, Georg Ertl ${ }^{1}$, Frank Weidemann ${ }^{3}$, Stefan \\ Frantz $^{1}$, Stefan Störk ${ }^{1}$, Peter Nordbeck ${ }^{1}$ \\ ${ }^{1}$ Department of Internal Medicine I, Comprehensive Heart Failure Center, University Hospital Würzburg, Würzburg, Germany; ${ }^{2}$ Department of \\ Internal Medicine II, University Hospital Würzburg, Würzburg, Germany; ${ }^{3}$ Medizinische Klinik I des Klinikum Vest, Recklinghausen, Germany \\ Contributions: (I) Conception and design: K Hu, P Nordbeck, F Weidemann, S Knop; (II) Administrative support: G Ertl, S Störk, S Frantz, P \\ Nordbeck; (III) Provision of study materials or patients: K Hu, D Liu; (IV) Collection and assembly of data: K Hu, D Liu, T Salinger, D Oder; (V) \\ Data analysis and interpretation: K Hu, D Liu; (VI) Manuscript writing: All authors; (VII) Final approval of manuscript: All authors. \\ \#These authors contributed equally to this work. \\ Correspondence to: Dr. Kai Hu; Dr. Peter Nordbeck. Department of Internal Medicine I, University Hospital Würzburg, and Comprehensive Heart \\ Failure Center, Oberdürrbacher Str. 6, 97080 Würzburg, Germany. Email: hu_k@ukw.de; nordbeck_p@ukw.de.
}

Background: This study aimed to explore the value of cardiac biomarker [serum high sensitive troponin T (hs-TNT) and N-terminal pro-brain natriuretic peptide (NT-proBNP)] measurement in the differential diagnosis of infiltrative cardiomyopathy patients [Friedreich's ataxia (FA), Fabry disease (FD) and light-chain (AL) cardiac amyloidosis (CA)] with preserved left ventricular (LV) systolic function.

Methods: Between 2012 and 2014, all consecutive patients presenting at our center with infiltrative cardiomyopathy and concomitant symmetrical LV hypertrophy as well as preserved LV systolic function were included in this study. Serum hs-TNT and NT-proBNP, morphologic and functional features derived from echocardiography and cardiac magnetic resonance imaging (cMRI) examinations were compared among these patients.

Results: A total of 57 patients (FA 20, FD 23 and CA 14) were included. Hs-TNT and NT-proBNP levels were significantly higher in the CA group [median: hs-TNT $98 \mathrm{pg} / \mathrm{mL}, \mathrm{NT}$-proBNP 4,110 pg/mL] than in the FA group [hs-TNT $14 \mathrm{pg} / \mathrm{mL}$, NT-proBNP $40 \mathrm{pg} / \mathrm{mL}$ ] and FD group [hs-TNT $18 \mathrm{pg} / \mathrm{mL}$, NTproBNP $131 \mathrm{pg} / \mathrm{mL}$, both $\mathrm{P}<0.001]$. There was a negative correlation between NT-proBNP and estimated glomerular filtration rate (eGFR) in CA patients $(\mathrm{r}=-0.72, \mathrm{P}=0.012)$. Both hs-TNT $>60 \mathrm{pg} / \mathrm{mL}$ (sensitivity 0.79 , specificity 0.93 ) and NT-proBNP $>1,000 \mathrm{pg} / \mathrm{mL}$ (sensitivity 0.91 , specificity 0.93 ) excellently differentiated CA from FA and FD.

Conclusions: Increased hs-TNT and NT-proBNP levels are suggestive of CA diagnosis among patients with infiltrative cardiomyopathy and preserved LV ejection fraction.

Keywords: Friedreich's ataxia; Fabry disease (FD); cardiac amyloidosis (CA); diagnosis

Submitted Jan 13, 2018. Accepted for publication Jul 10, 2018.

doi: $10.21037 /$ jtd.2018.07.56

View this article at: http://dx.doi.org/10.21037/jtd.2018.07.56

\section{Introduction}

Several infiltrative cardiomyopathies, as in the case of cardiac amyloidosis (CA), Friedreich's ataxia (FA) and Fabry disease (FD), mimic hypertrophic or hypertensive cardiomyopathy (1). Since all these conditions are rare and also characterized by variable physiologic and morphologic characteristics, the risk of misdiagnosis is high (1). A comprehensive diagnostic strategy including ECG, cardiac 
imaging, genetic testing, urine test (for example, urinary globotriaosylsphingosine in FD) is of importance to establish the correct diagnosis prior to the initiation of the appropriate therapy for these patients (2). However, not only the diagnosis, but also the staging of disease severity as well as the monitoring of treatment response remain problematic in infiltrative cardiomyopathies. It is known that measurements of cardiac biomarkers might add valuable additional information for the diagnosis, staging and evaluating the therapy responses for various cardiovascular diseases. The diagnostic and prognostic value of cardiac biomarkers such as cardiac troponin T (TNT) and brain natriuretic peptide (BNP) in patients with myocardial infarction and chronic heart failure has been extensively studied $(3,4)$. Increased BNP and high-sensitivity TNT (hs-TNT) levels were found not only in heart failure patients with reduced, but also those with preserved left ventricular ejection fraction (LVEF) $(5,6)$.

The diagnostic and prognostic value of BNP and NTproBNP as well as TNT in CA patients has been described in previous studies $(7,8)$. Moreover, Coats and colleagues demonstrated that NT-proBNP concentrations are also elevated in patients with FD in case of early cardiac involvement (9). Recently, we showed that hs-TNT is a useful biomarker for staging FD patients (10). However, there is only scarce information on the role of BNP and TNT in FA patients, despite the fact that heart failure serves as a major complication of $\mathrm{FA}$ and heart failure is the primary mode of death in $\sim 60 \%$ of patients with FA (11). Accordingly, our group showed that a threshold for hs-TNT $\geq 14 \mathrm{pg} / \mathrm{mL}$ could be used to identify severe cardiomyopathic involvement in FA patients (12). The present study aimed to compare hs-TNT and NT-proBNP values in CA, FA and FD patients with preserved LVEF and tested the hypothesis that the increase in hs-TNT and NT-proBNP values might be more pronounced in $\mathrm{CA}$ as compared to FA and FD patients with preserved LVEF, thus, increased hs-TNT and NT-proBNP values might be suggestive of CA diagnosis among infiltrative cardiomyopathy patients with concomitant symmetrical LV hypertrophy and preserved LV systolic function.

\section{Methods}

\section{Study population}

Consecutive FA ( $\mathrm{n}=20), \mathrm{FD}(\mathrm{n}=23)$ and light-chain (AL) CA $(n=14)$ patients with preserved LVEF were included in this study. Data of standard echocardiography, cardiac magnetic resonance imaging (cMRI), serum NT-proBNP and hs-TNT concentrations were analyzed. Echocardiographic enrollment criteria for further analysis included: (I) $\mathrm{LV}$ hypertrophy, i.e., $\mathrm{LV}$ wall thickness $>10 \mathrm{~mm}$ in $\mathrm{FA},>11 \mathrm{~mm}$ in $\mathrm{FD}$, and $>12 \mathrm{~mm}$ in $\mathrm{CA}$; and (II) LVEF $\geq 55 \%$. Patients with coronary artery disease, moderate or severe valvular heart disease, and other endocrine or systemic disease were excluded, as well as patients presenting with end-stage renal dysfunction [estimated glomerular filtration rate (eGFR) $<15 \mathrm{~mL} / \mathrm{min} / 1.73 \mathrm{~m}^{2}$ ]. FA and FD were genetically confirmed in all patients. Amyloid was confirmed histologically with at least one tissue biopsy (endomyocardial tissue, bone marrow, rectum, kidney, or subcutaneous fat). The type of amyloid was assessed by immune histology. Cardiac involvement was evidenced by either an endomyocardial biopsy demonstrating amyloidosis in the presence of clinical or laboratory evidence of involvement, or echocardiographic evidence of amyloidosis in patients tested positive in noncardiac biopsies, based on the current international consensus $(13,14)$. Echocardiographic features of cardiac involvement in amyloidosis included a mean LV wall thickness greater than $12 \mathrm{~mm}$ in the absence of hypertension or other potential causes of LV hypertrophy (14).

\section{Hs-TNT and NT-proBNP measurements}

Peripheral venous blood was taken and collected in serum tubes. Fresh blood samples were immediately centrifuged at 5,000× U/min for 10 minutes and forwarded to analysis within 1 hour. Serum hs-TNT and NTproBNP concentrations were measured in the laboratory of the University Hospital Würzburg using the novel electrochemiluminescence technology on a Roche Cobas e411 analyzer.

\section{Echocardiography}

Echocardiography was performed on a Vivid 7 or E9 scanner (GE, Horten, Norway) and standard 2D gray scale views, pulsed- and continuous-wave Doppler as well as tissue Doppler imaging were recorded. Off-line measurements were performed by one single investigator (DL) as described previously (15). End-diastolic LV dimension (LVEDD), as well as thickness of the septum (IVSd) and the posterior wall (PWd), as well as end-systolic left atrial diameter were measured in parasternal LV long axis views. LVEF was measured with the biplane Simpson 
method in the apical 4- and 2-chamber views. Diastolic function parameters included early- and late-diastolic mitral inflow velocities (E-wave and A-wave), E/A ratio, and deceleration time (DT) of E-wave. Tissue Doppler derived early-diastolic mitral annular velocity (E') of the septal annulus was acquired in the apical 4-chamber view.

\section{Two-dimensional speckle tracking imaging (2D-STI)}

Global and segmental longitudinal systolic strain assessments were performed off-line using dedicated software as previously described (EchoPAC, GE, Horten, Norway) (15). LV longitudinal peak systolic strain $\left(\mathrm{LS}_{\mathrm{sy}}\right)$ was extracted from basal, mid, and apical segments of the septum. The ratio between apical and basal septal $\mathrm{LS}_{\mathrm{sys}}$ (LSsys api/bas) was calculated as apical septal $\mathrm{LS}_{\text {sys }}$ divided by basal septal $\mathrm{LS}_{\text {sys }}$.

\section{Cardiac cMRI}

Cardiac MRI with late enhancement (LE) imaging was performed on a 1.5 Tesla whole-body scanner (Magnetom Symphony Quantum, Siemens Healthcare, Erlangen, Germany), using two conventional six-channel body phased-array coils for signal detection. A stack of 15 slices assured coverage of the whole LV. LE was obtained 12-15 minutes after the injection of gadopentetate dimeglumine $(0.2 \mathrm{mmol} / \mathrm{kg})$ using an inversion recovery $2 \mathrm{D}$ turbo-gradient echo pulse sequence.

\section{Statistical analysis}

Statistical analysis was performed using IBM SPSS, version 23 for Windows (IBM Corp., New York, USA). A $\mathrm{P}$ value $<0.05$ (two-tailed test) was considered statistically significant. Continuous variables are expressed as mean \pm standard deviation (SD) or median (quartiles), and categorical variables as count (percent). Differences on continuous data among the three patient groups were compared using one-way analysis of variance (ANOVA) after normalization if indicated, followed by appropriate post-hoc tests for multiple comparisons (Tukey's or Games-Howell). Non-normally distributed variables were normalized prior to analysis using natural logarithm values (ln). Categorical data were compared using a chi-square test for the overall test and Fisher's exact for pair-wise group tests.

Pearson's correlation coefficient was used to assess linear dependence between the biomarkers and other related variables. Receiver operating characteristic (ROC) analysis was performed to identify the diagnostic performance of biomarkers hs-TNT and NT-proBNP and other parameters for distinguishing $\mathrm{CA}$ from $\mathrm{FA}$ and FD, respectively. The cutoff values were identified from ROC analysis by maximizing the sum of the sensitivity and specificity.

\section{Results}

\section{Clinical characteristics and echocardiographic and cMRI measurements}

As shown in Table 1, patients were significantly older in the CA group than in the FA and FD groups. The majority of CA patients were in NYHA functional class II (71.4\%), whereas most FA and FD patients were in class I (FA $80.0 \%$ and FD $60.9 \%)$. A total of 11 (78.6\%) patients with CA received chemotherapy or/and stem cell transplant therapy (9 with chemotherapy alone and 2 received high dose chemotherapy plus stem cell transplant therapy); 15 (65.2\%) patients with FD received enzyme replacement therapy. There was no patient treated with immunomodulator therapy, nor either of the novel treatment options for FD—such as migalastat or lucerastat $(16,17)$-in this patient cohort.

Table 1 Clinical, echocardiographic and cardiac magnetic resonance imaging characteristics in different infiltrative cardiomyopathies

\begin{tabular}{lcccc}
\hline Variables & $\mathrm{FA}(\mathrm{n}=20)$ & $\mathrm{FD}(\mathrm{n}=23)$ & $\mathrm{CA}(\mathrm{n}=14)$ & $\mathrm{P}$ value \\
\hline Age (years) & $33 \pm 14$ & $42 \pm 14$ & $66 \pm 9^{\star \dagger}$ & $<0.001$ \\
Age range (years) & $19-68$ & $20-68$ & $48-83$ & $<0.001$ \\
Male [n (\%)] & $14(70.0)$ & $10(43.5)$ & $9(64.3)$ & 0.183 \\
BMI $\left(\mathrm{kg} / \mathrm{m}^{2}\right)$ & $24 \pm 4$ & $25 \pm 6$ & $25 \pm 5$ & 0.649 \\
\hline
\end{tabular}

Table 1 (continued) 
Table 1 (continued)

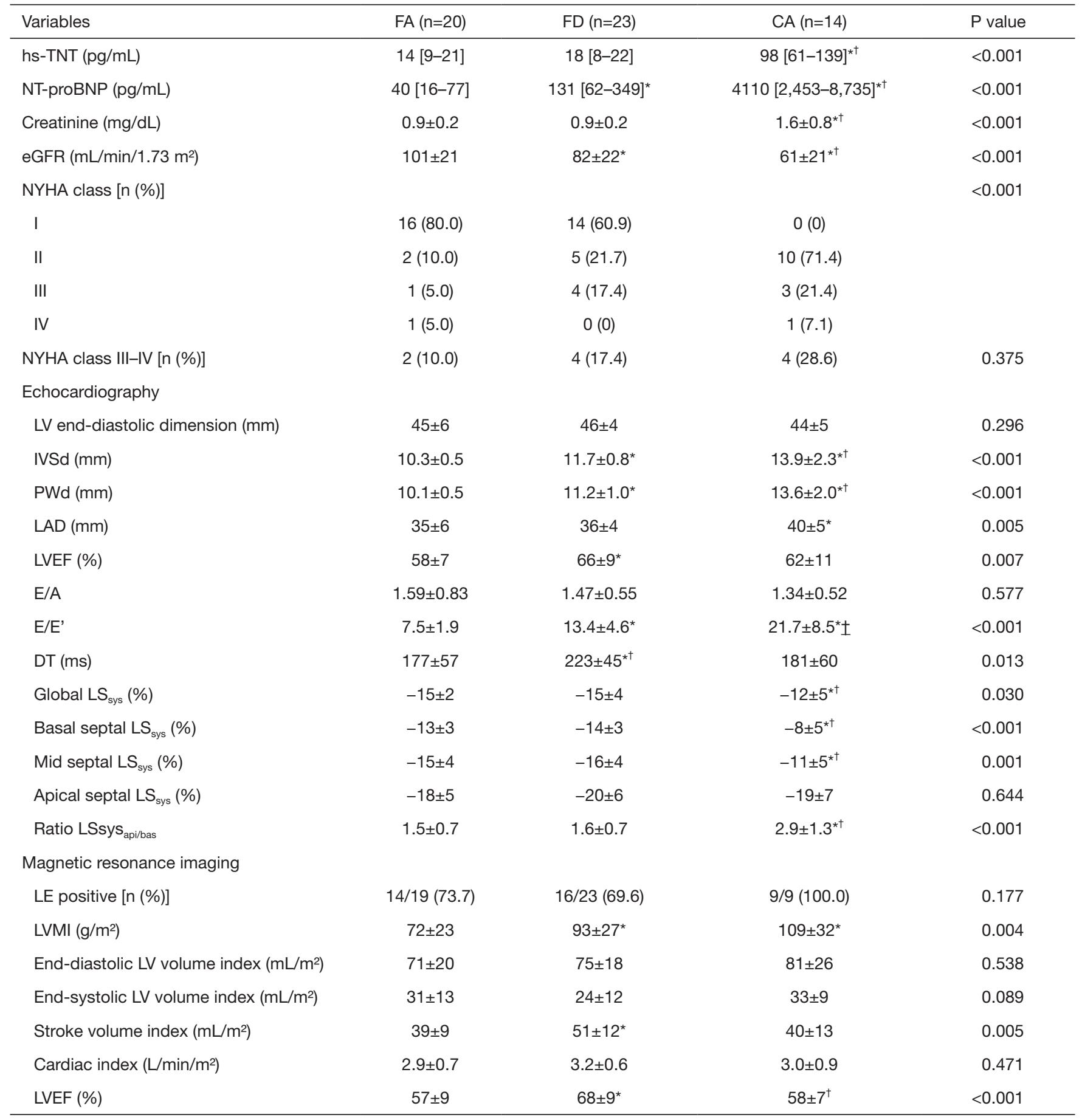

*, $\mathrm{P}<0.05$ vs. $\mathrm{FA} ;{ }^{\dagger}, \mathrm{P}<0.05$ vs. FD. FA, Friedreich' Ataxia; FD, Fabry disease; CA, light-chain cardiac amyloidosis; BMI, body mass index; hs-TNT, high sensitive troponin T; NT-proBNP, N-terminal pro-brain natriuretic peptide; eGFR, estimated glomerular filtration rate; NYHA, New York Heart Association; LV, left ventricular; LVEDD, left ventricular end-diastolic dimension; IVSd, interventricular septal end-diastolic wall thickness; PWd, posterior wall thickness; LAD, left atrium anteroposterior diameter; LVEF, left ventricular ejection fraction; E/A, early (E-wave) to late (A-wave) mitral inflow velocity ratio; DT, E-wave deceleration time; E', Tissue Doppler derived early-diastolic mitral annular

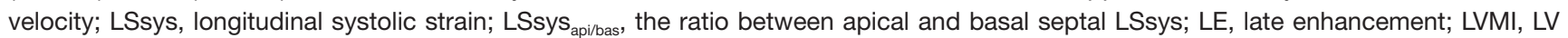
mass indexed to body surface area. 

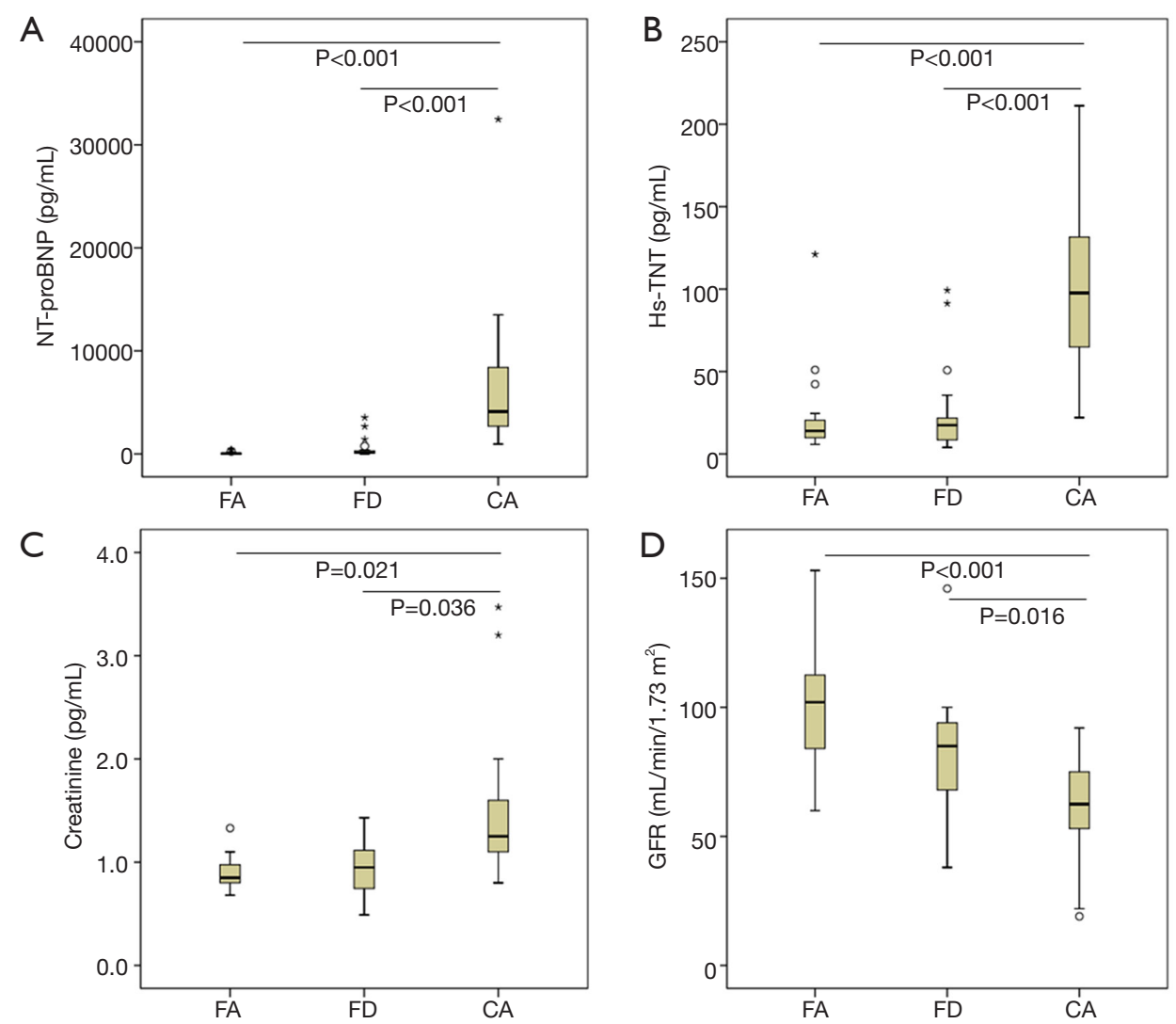

Figure 1 Serum NT-proBNP (A), hs-TNT (B), creatinine (C), and estimated GFR (D) in different infiltrative cardiomyopathies. eGFR, estimated glomerular filtration rate; FA, Friedreich's Ataxia; FD, Fabry disease; CA, light-chain cardiac amyloidosis; hs-TNT, high sensitive troponin T; NT-proBNP, N-terminal pro-brain natriuretic peptide.

LV hypertrophy (IVSd and PWd) was more significant and left atria tended to be larger in CA patients compared to FA and FD patients. E/E' was also significantly higher in CA patients than in FA and FD patients, and significantly higher in FD patients compared to FA patients. Of note, 2D-STI derived global longitudinal systolic strain, septal basal and mid $L_{\text {sys }}$ were all significantly lower in CA patients compared to FA and FD patients. As expected, LSsys $_{\text {apibas }}$ ratio was significantly higher in CA patients than in FA and FD patients. LE was evidenced by $\mathrm{cMRI}$ in $74 \%$ of FA, $70 \%$ of FD and $100 \%$ of CA patients. cMRI detected $\mathrm{LV}$ mass index was also significantly higher in the CA group compared to the FA group and also in the FD group compared to the FA group.

As shown in Figure $1 \mathrm{~A}$, serum NT-proBNP level was significantly higher in $\mathrm{CA}$ patients than in FA and FD patients (both $\mathrm{P}<0.001)$. Similarly, hs-TNT level was significantly higher in CA patients than in FA and FD patients (Figure 1B). Moreover, serum creatinine was higher and eGFR was significantly lower in CA patients compared to FA and FD patients (Figure 1C,D).

\section{Correlation of hs-TNT and NT-proBNP with other parameters in different infiltrative cardiomyopathies}

Table 2 shows the correlation results of hs-TNT and NTproBNP with other clinical and laboratory parameters in patients with CA. NT-proBNP but not hs-TNT was negatively correlated with eGFR in CA patients $(r=-0.724$, $\mathrm{P}=0.012$, Figure 2).

\section{Diagnostic performance of NT-proBNP and bs-TNT among infiltrative cardiomyopathy patients with symmetrical hypertrophy and preserved LVEF}

NT-proBNP and hs-TNT yielded excellent diagnostic 
Table 2 Pearson's correlation coefficients between NT-proBNP and hs-TNT and other parameters in patients with light-chain cardiac amyloidosis

\begin{tabular}{|c|c|c|c|c|}
\hline \multirow{2}{*}{ Variables } & \multicolumn{2}{|c|}{ NT-proBNP } & \multicolumn{2}{|c|}{ Hs-TNT } \\
\hline & $\mathrm{n}$ & $P$ value & $\mathrm{n}$ & $P$ value \\
\hline Age & -1.116 & ns & -0.001 & ns \\
\hline eGFR & -0.724 & 0.012 & -0.223 & ns \\
\hline Hs-TNT & 0.129 & ns & 1 & - \\
\hline NT-proBNP & 1 & - & 0.129 & ns \\
\hline IVSd (mm) & -0.077 & ns & -0.351 & ns \\
\hline LVEF (\%) & 0.061 & ns & 0.230 & ns \\
\hline LAD (mm) & 0.215 & ns & 0.351 & ns \\
\hline E/E' & 0.190 & ns & -0.090 & ns \\
\hline GLS (\%) & -0.132 & ns & -0.256 & ns \\
\hline MRI-LVMI $\left(\mathrm{g} / \mathrm{m}^{2}\right)$ & 0.040 & ns & -0.195 & ns \\
\hline
\end{tabular}

hs-TNT, high sensitive troponin T; NT-proBNP, N-terminal pro-brain natriuretic peptide; eGFR, estimated glomerular filtration rate; LAD, left atrium anteroposterior diameter; LVEF, left ventricular ejection fraction; E', Tissue Doppler derived early-diastolic mitral annular velocity; LVMI, LV mass indexed to body surface area; GLS, global longitudinal systolic strain; MRI, magnetic resonance imaging; ns, not significant.

\section{A}

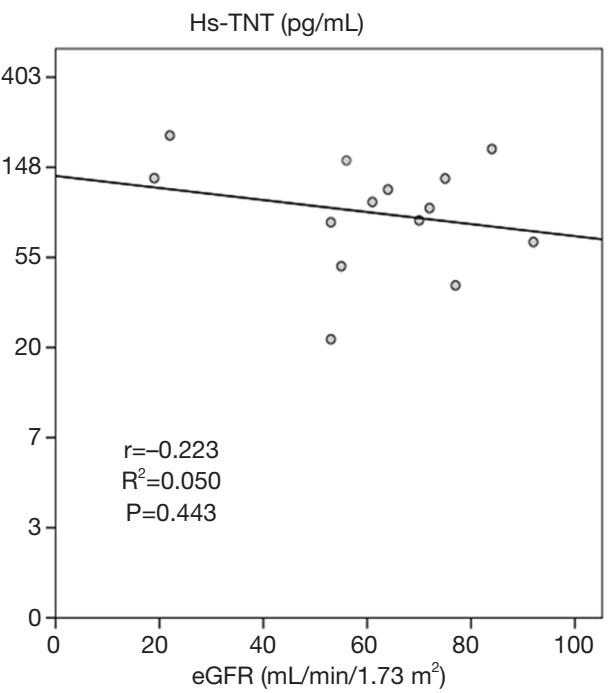

power for differentiating CA from FA and FD with an AUC of 0.98 and 0.93 , respectively (Figure 3). Table 3 demonstrates the performance of various laboratory and imaging markers for the diagnosis of CA. Hs-TNT $>60 \mathrm{pg} / \mathrm{mL}$, NT-proBNP $>1,000 \mathrm{pg} / \mathrm{mL}, \mathrm{E} / \mathrm{E}^{\prime}>12$, LSsys $_{\text {api } / \text { as }}$ ratio $>2.0$, hs-TNT $>60 \mathrm{pg} / \mathrm{mL}+$ NT-proBNP $>1,000 \mathrm{pg} / \mathrm{mL}$, hs-TNT $>60 \mathrm{pg} / \mathrm{mL}+$ E/E' $>12$, hs-TNT $>60 \mathrm{pg} / \mathrm{mL}+\mathrm{LSsys}_{\text {api/bas }}$ ratio $>2.0$ yielded satisfactory diagnostic performance for CA. The best diagnostic performance was found for hs-TNT $>60 \mathrm{pg} / \mathrm{mL}$ (sensitivity 0.79 , specificity 0.93 ) and NT-proBNP $>1,000 \mathrm{pg} / \mathrm{mL}$ (sensitivity 0.91 , specificity 0.93 ).

\section{Discussion}

The major finding of present study is that a profound increase of serum NT-proBNP and hs-TNT is evidenced in CA patients with preserved LVEF, whereas these biomarkers are usually only mildly increased in FD patients with preserved LVEF, and remain largely in the normal range in FA patients with preserved LVEF. Increased NT-proBNP $(>1,000 \mathrm{pg} / \mathrm{mL})$ and hs-TNT $(>60 \mathrm{pg} / \mathrm{mL})$ levels, which were rarely present in FD and FA, might thus

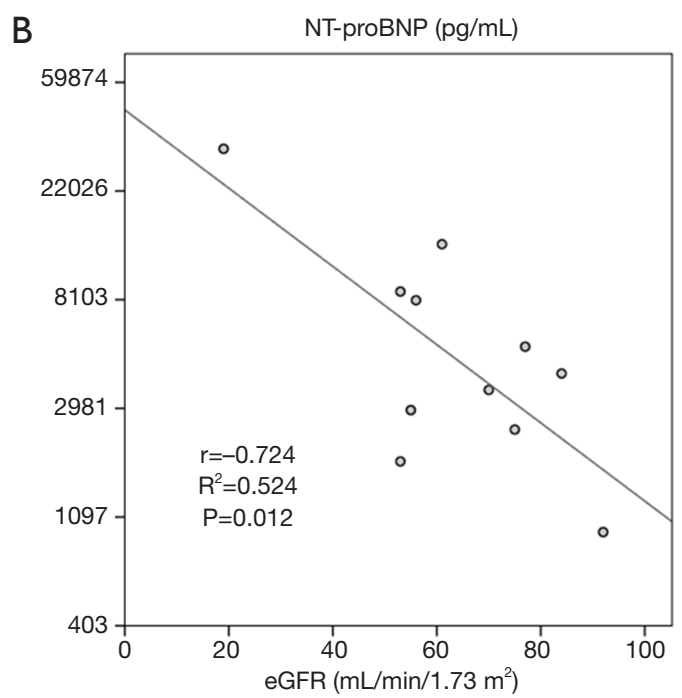

Figure 2 Linear correlations between hs-TNT and NT-ProBNP and eGRF in patients with CA. eGFR, estimated glomerular filtration rate; CA, light-chain cardiac amyloidosis; hs-TNT, high sensitive troponin T; NT-proBNP, N-terminal pro-brain natriuretic peptide. 
help to aid the initial diagnosis of CA among infiltrative cardiomyopathy patients with symmetrical hypertrophy and preserved LVEF.

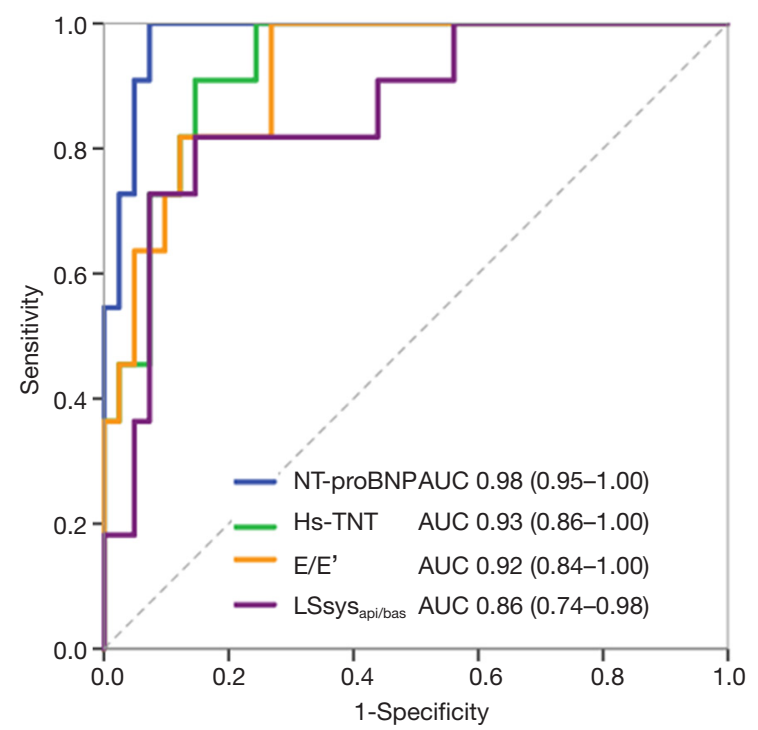

Figure 3 ROC curves of hs-TNT, NT-proBNP, and other echocardiographic markers (E/E' and $\mathrm{LSsys}_{\text {api/bas }}$ ) for differentiating CA from FA and FD patients. E/E', the ratio between mitral inflow velocity and mitral annular velocity at early-diastole; LSsys api/ bas, the ratio between apical and basal septal longitudinal systolic strain; FA, Friedreich's Ataxia; FD, Fabry disease; CA, light-chain cardiac amyloidosis; ROC, receiver operating characteristic; hsTNT, high sensitive troponin T; NT-proBNP, N-terminal probrain natriuretic peptide.

\section{Increased NT-proBNP and bs-TNT levels in CA patients with preserved $L V E F$}

CA could be diagnosed either directly by endomyocardial biopsy or indirectly by non-invasive diagnostic modalities, such as standard echocardiography and 2D-STI derived apical sparing of longitudinal strain $(12,18)$, cMRI, ECG, and histological confirmation of amyloid in a non-cardiac tissue specimen (1). In line with previous findings $(3,6)$, the present study showed that both NT-proBNP and hsTNT levels were significantly increased in CA patients with preserved LVEF.

A previous study has described that heart dysfunction and renal failure were the two determinants of the concentration of natriuretic peptides in patients with amyloidosis (19). Accordingly, enrolled CA patients in this study presented both subclinical systolic dysfunction (reduced longitudinal strain values but normal LVEF), diastolic dysfunction (increased E/E' value), and reduced renal function (increased creatinine level and reduced eGFR value). These characteristics thus contributed to the increased NT-proBNP profile found in CA patients with preserved LVEF in our study. It is thus not surprising that higher NT-proBNP correlated with a reduced eGFR in the CA patients with preserved LVEF in this study. Serum cardiac biomarkers provide a quantitative assessment of cardiac damage (troponin I or T) and wall strain (BNP, NT-proBNP), which are the most important outcome predictors in AL amyloidosis. From a diagnostic point of view, values of NT-proBNP levels may be more useful as a negative predictive marker rather than indicator of cardiac

Table 3 Diagnostic performance of NT-proBNP and hs-TNT for differentiating CA from FA and FD

\begin{tabular}{lcccc}
\hline Variables & $\begin{array}{c}\text { Sensitivity } \\
(95 \% \mathrm{Cl})\end{array}$ & $\begin{array}{c}\text { Specificity } \\
(95 \% \mathrm{Cl})\end{array}$ & $\begin{array}{c}\text { Positive predictive } \\
\text { value }(95 \% \mathrm{Cl})\end{array}$ & $\begin{array}{c}\text { Negative predictive } \\
\text { value }(95 \% \mathrm{Cl})\end{array}$ \\
\hline Hs-TNT $>60 \mathrm{pg} / \mathrm{mL}$ & $0.79(0.49-0.94)$ & $0.93(0.80-0.98)$ & $0.79(0.49-0.94)$ & $0.93(0.80-0.98)$ \\
NT-proBNP $>1,000 \mathrm{pg} / \mathrm{mL}$ & $0.91(0.57-1.00)$ & $0.93(0.79-0.98)$ & $0.77(0.46-0.94)$ & $0.98(0.85-1.00)$ \\
E/E' $>12$ & $1.00(0.73-1.00)$ & $0.67(0.51-0.80)$ & $0.50(0.31-0.69)$ & $1.00(0.85-1.00)$ \\
Ratio LSsys ${ }_{\text {api/bas }}>2.0$ & $0.79(0.49-0.94)$ & $0.83(0.68-0.92)$ & $0.61(0.36-0.82)$ & $0.92(0.78-0.98)$ \\
Hs-TNT $>60 \mathrm{pg} / \mathrm{mL}+$ NT-proBNP $>1,000 \mathrm{pg} / \mathrm{mL}$ & $0.64(0.32-0.88)$ & $0.98(0.86-1.00)$ & $0.88(0.47-0.99)$ & $0.91(0.78-0.97)$ \\
Hs-TNT $>60 \mathrm{pg} / \mathrm{mL}+$ E/E' $>1$ & $0.71(0.42-0.90)$ & $0.98(0.86-1.00)$ & $0.91(0.57-1.00)$ & $0.91(0.78-0.97)$ \\
Hs-TNT $>60 \mathrm{pg} / \mathrm{mL}+$ ratio LSsys & $0.57(0.30-0.81)$ & $0.98(0.86-1.00)$ & $0.89(0.51-0.99)$ & $0.87(0.74-0.95)$ \\
\hline
\end{tabular}

FA, Friedreich' Ataxia; FD, Fabry disease; CA, light-chain cardiac amyloidosis; hs-TNT, high sensitive troponin T; NT-proBNP, N-terminal pro-brain natriuretic peptide; E', Tissue Doppler derived early-diastolic mitral annular velocity; LSsys, longitudinal systolic strain; LSsys $_{\text {apibas }}$, the ratio between apical and basal septal LSsys; Cl, confidence interval. 
involvement in the course of AL amyloidosis. In prior studies, myocardial amyloid was shown excluded by normal values of NT-proBNP, and no patient with cardiac AL was found to have a NT-proBNP of $<55 \mathrm{pmol} / \mathrm{L}$ (7). It is to note that CA patients were significantly older than FA and FD patients in the current study, since age is related to higher NT-proBNP values (20), the increased NT-proBNP profile found in CA patients with preserved LVEF in our study might partly be related to aging. However, the significantly increased extent of NT-proBNP values in CA patients as compared with FA and FD patients by far exceeded the impact attributable of aging in studied patients.

Cardiac troponins T (cTNT) and I (cTNI) are highly specific and sensitive markers of cardiac injury including subtle myocyte damage (21). Increased cTNT was reported to be increased and related to prognosis of CA patients (22). In line with this finding, we could show increased hs-TNT in CA patients with preserved LVEF, reflecting the chronic cardiac injury in CA. Our study also demonstrated the cardiac specificity of hs-TNT increase in CA patients since this parameter was not correlated to reduced eGFR in CA patients, this observation suggests that the severity of renal and cardiac involvement might not always run in parallel in individual CA patient.

The possible underlying pathophysiological mechanisms for predominantly increased cardiac biomarker levels in patients with CA compared to patients with other disease entities remain obscure. Molecular evidence has demonstrated that cardiac damage and rapid progression of heart failure in $\mathrm{AL}$ amyloidosis may be partially related to a significant specific cardiotoxic effect of an AL amyloidosis precursor in the circulation than the direct infiltrative damage by amyloid fibrils in the myocardial tissue. Furthermore, the toxic damage induced by these precursors seems to be capable to directly stimulate the release of NTproBNP from ventricular myocardial cells (21-24).

\section{NT-proBNP and bs-TNT levels in FA and FD patients with preserved LVEF}

In the present study, NT-proBNP and hs-TNT levels were slightly increased in FD patients and remained largely normal in FA patients in case of preserved LVEF. For enrolled FD patients in this study, the slightly increased NT-proBNP values could be partly explained by the mildly reduced kidney function. Regarding cardiac involvement, the mean value of hs-TNT was about $14 \mathrm{pg} / \mathrm{mL}$ in FD patients, since $>14 \mathrm{pg} / \mathrm{mL}$ has been defined as cutoff value for staging cardiomyopathy in FD patients in a previous study (10). FD patients included in the current study might be defined in the stage of intermediate cardiomyopathy. It is to note that the hs-TNT levels in our FD patient cohort are somehow lower than previously reported $(10,25)$. The main underlying reason might be that FD patients probably were in a more advanced disease stage in the previous reports than patients included in the current FD patient cohort, since only patients with preserved left ventricular (LF) ejection fraction were included in the current study, therefore excluding many patients with end-stage FD cardiomyopathy. In fact, LE was detected by cMRI in $70 \%$ of FD patients despite preserved LVEF and slightly reduced longitudinal strain values. Moreover, increased E/ E' (reflecting diastolic dysfunction) values were evidenced in these patients. Thus, slightly elevated NT-proBNP and hs-TNT levels could be used to define the intermediate cardiomyopathy stage of FD patients with preserved LVEF.

The development of a severe cardiomyopathy in patients with FA, usually leading to death from congestive heart failure in the third to fifth decade of life has been described before (23). The mean age in the current patient cohort was 33 years old [range 19 to 68 years, with 11 (55\%) younger than 30 years]. It can thus be anticipated that many enrolled patients were in a very early stage of cardiac involvement, if any. Accordingly, there were no signs of kidney dysfunction (normal creatinine and eGFR values) in these FA patients, although, there were signs of early subtle LV systolic dysfunction reflected by slightly reduced longitudinal strain values in these patients. Thus, it is not strange that normal NT-proBNP and hs-TNT values were found in enrolled FA patients with preserved LVEF in our study.

\section{Conclusions}

Increased hs-TNT and NT-proBNP levels are suggestive of CA diagnosis among patients with infiltrative cardiomyopathy and preserved $\mathrm{LV}$ ejection fraction.

\section{Limitations}

Caution is needed in interpreting the study results due to the small patient sample size of our study, which might be linked with data selection bias. Prospective studies with larger sample size are warranted to validate these results. Due to the small patient sample, further subdividing patients with various degrees of kidney dysfunction according to eGFR was not practicable. The study results might also be affected 
by various therapy regimens applied to included patients, especially in FD and CA patients. Thus, larger studies observing patients under various therapy strategies as well as long-term follow-up observations on the natural history and prognostic impact of cardiac biomarkers in CA, FD, and especially in FA patients are warranted. Another study limitation of the present study is the lack of data regarding non-contrast T1 mapping derived from cMRI examinations. Non-contrast T1 mapping has recently shown exceptional potential as a unique and powerful diagnostic tool in the imaging assessment of LV hypertrophy and FD (24).

\section{Clinical implications}

Besides ECG, non-invasive cardiac imaging modalities including echocardiography and cMRI, endomyocardial biopsy, as well as histological confirmation of amyloid in non-cardiac tissue, determination of cardiac biomarkers might add to the diagnostic precision in identification of CA patients with preserved LVEF. In the setting of routine echocardiographic examinations, it is necessary to immediately speculate on the etiology of patients presenting with LV hypertrophy as the common result of several different disease entities. Echocardiographic examination in conjunction with routine biomarkers could help to select subsequent specific examinations right away to allow making the final diagnosis of the underlying disease in patients with LF hypertrophy. Today, patients with CA and FD are suitable for a still increasing large spectrum of disease-specific therapies able to improve outcomes substantially.

\section{Acknowledgements}

Funding: This work was supported by grants from the Bundesministerium für Bildung und Forschung (BMBF 01EO1004 and 01EO1504).

\section{Footnote}

Conflicts of Interest: The authors have no conflicts of interest to declare.

Ethical Statement: The study was approved by Local Ethics Committee at the University of Würzburg and conducted in accordance to the Declaration of Helsinki. Written informed consent was obtained from the patients or their guardians.

\section{References}

1. Seward JB, Casaclang-Verzosa G. Infiltrative cardiovascular diseases: cardiomyopathies that look alike. J Am Coll Cardiol 2010;55:1769-79.

2. Weidemann F, Ertl G, Wanner C, et al. The Fabry cardiomyopathy - diagnostic approach and current treatment. Curr Pharm Des 2015;21:473-8.

3. Vuolteenaho O, Ala-Kopsala M, Ruskoaho H. BNP as a biomarker in heart disease. Adv Clin Chem 2005;40:1-36.

4. Spratt DP, Mellanby RJ, Drury N, et al. Cardiac troponin I: evaluation I of a biomarker for the diagnosis of heart disease in the dog. J Small Anim Pract 2005;46:139-45.

5. Tate S, Griem A, Durbin-Johnson B, et al. Marked elevation of B-type natriuretic peptide in patients with heart failure and preserved ejection fraction. J Biomed Res 2014;28:255-61.

6. Jhund PS, Claggett BL, Voors AA, et al. Elevation in high-sensitivity troponin $\mathrm{T}$ in heart failure and preserved ejection fraction and influence of treatment with the angiotensin receptor neprilysin inhibitor LCZ696. Circ Heart Fail 2014;7:953-9.

7. Palladini G, Campana C, Klersy C, et al. Serum N-terminal pro-brain natriuretic peptide is a sensitive marker of myocardial dysfunction in AL amyloidosis. Circulation 2003;107:2440-5.

8. Dispenzieri A, Gertz MA, Kyle RA, et al. Serum cardiac troponins and $\mathrm{N}$-terminal pro-brain natriuretic peptide: a staging system for primary systemic amyloidosis. J Clin Oncol 2004;22:3751-7.

9. Coats CJ, Parisi V, Ramos M, et al. Role of serum $\mathrm{N}$-terminal pro-brain natriuretic peptide measurement in diagnosis of cardiac involvement in patients with andersonfabry disease. Am J Cardiol 2013;111:111-7.

10. Seydelmann N, Liu D, Kramer J, et al. High-Sensitivity Troponin: A Clinical Blood Biomarker for Staging Cardiomyopathy in Fabry Disease. J Am Heart Assoc 2016;5:e02839.

11. Payne RM, Wagner GR. Cardiomyopathy in Friedreich ataxia: clinical findings and research. J Child Neurol 2012;27:1179-86.

12. Hu K, Liu D, Nordbeck P, et al. Impact of monitoring longitudinal systolic strain changes during serial echocardiography on outcome in patients with $\mathrm{AL}$ amyloidosis. Int J Cardiovasc Imaging 2015;31:1401-12.

13. Gertz MA, Merlini G. Definition of organ involvement and response to treatment in AL amyloidosis: an updated consensus opinion. Amyloid 2010;17:48-9. 
14. Gertz MA, Comenzo R, Falk RH, et al. Definition of organ involvement and treatment response in immunoglobulin light chain amyloidosis (AL): a consensus opinion from the 10th International Symposium on Amyloid and Amyloidosis, Tours, France, 18-22 April 2004. Am J Hematol 2005;79:319-28.

15. Liu D, Hu K, Niemann M, et al. Effect of combined systolic and diastolic functional parameter assessment for differentiation of cardiac amyloidosis from other causes of concentric left ventricular hypertrophy. Circ Cardiovasc Imaging 2013;6:1066-72.

16. Muntze J, Salinger T, Gensler D, et al. Treatment of hypertrophic cardiomyopathy caused by cardiospecific variants of Fabry disease with chaperone therapy. Eur Heart J 2018;39:1861-2.

17. Guerard N, Oder D, Nordbeck P, et al. Lucerastat, an Iminosugar for Substrate Reduction Therapy: Tolerability, Pharmacodynamics, and Pharmacokinetics in Patients With Fabry Disease on Enzyme Replacement. Clin Pharmacol Ther 2018;103:703-11.

18. Phelan D, Collier P, Thavendiranathan $\mathrm{P}$, et al. Relative apical sparing of longitudinal strain using two-dimensional speckle-tracking echocardiography is both sensitive and specific for the diagnosis of cardiac amyloidosis. Heart

Cite this article as: $\mathrm{Hu} \mathrm{K}$, Liu D, Salinger T, Oder D, Knop S, Ertl G, Weidemann F, Frantz S, Störk S, Nordbeck P. Value of cardiac biomarker measurement in the differential diagnosis of infiltrative cardiomyopathy patients with preserved left ventricular systolic function. J Thorac Dis 2018;10(8):49664975. doi: $10.21037 / j t d .2018 .07 .56$
2012;98:1442-8.

19. Palladini G, Foli A, Milani P, et al. Best use of cardiac biomarkers in patients with $\mathrm{AL}$ amyloidosis and renal failure. Am J Hematol 2012;87:465-71.

20. Raymond I, Groenning BA, Hildebrandt PR, et al. The influence of age, sex and other variables on the plasma level of $\mathrm{N}$-terminal pro brain natriuretic peptide in a large sample of the general population. Heart 2003;89:745-51.

21. Jaffe AS, Ravkilde J, Roberts R, et al. It's time for a change to a troponin standard. Circulation 2000;102:1216-20.

22. Dispenzieri A, Kyle RA, Gertz MA, et al. Survival in patients with primary systemic amyloidosis and raised serum cardiac troponins. Lancet 2003;361:1787-9.

23. Patel PI, Isaya G. Friedreich ataxia: from GAA tripletrepeat expansion to frataxin deficiency. Am J Hum Genet 2001;69:15-24.

24. Sado DM, White SK, Piechnik SK, et al. Identification and assessment of Anderson-Fabry disease by cardiovascular magnetic resonance noncontrast myocardial T1 mapping. Circ Cardiovasc Imaging 2013;6:392-8.

25. Kubo T, Baba Y, Hirota T, et al. Differentiation of infiltrative cardiomyopathy from hypertrophic cardiomyopathy using high-sensitivity cardiac troponin T: a case-control study. BMC Cardiovasc Disord 2015;15:53. 